Article

\title{
Investigation of an Optical Imaging Platform Integrated with an Ultrasound Application System for In Vitro Verification of Ultrasound-Mediated Drug Delivery
}

\author{
Jong-ryul Choi $\mathbb{1}$ and Juyoung Park* \\ Medical Device Development Center, Daegu-Gyeongbuk Medical Innovation Foundation (DGMIF), \\ Daegu 41061, Korea; jongryul32@dgmif.re.kr \\ * Correspondence: jyp@dgmif.re.kr; Tel.: +82-53-790-5590
}

Citation: Choi, J.-r.; Park, J.

Investigation of an Optical Imaging

Platform Integrated with an

Ultrasound Application System for In Vitro Verification of

Ultrasound-Mediated Drug Delivery. Appl. Sci. 2021, 11, 2846. https://

doi.org/10.3390/app11062846

Academic Editor: Francesco Dell'Olio

Received: 9 February 2021

Accepted: 18 March 2021

Published: 22 March 2021

Publisher's Note: MDPI stays neutral with regard to jurisdictional claims in published maps and institutional affiliations.

Copyright: (c) 2021 by the authors. Licensee MDPI, Basel, Switzerland. This article is an open access article distributed under the terms and conditions of the Creative Commons Attribution (CC BY) license (https:// creativecommons.org/licenses/by/ $4.0 /)$.

\begin{abstract}
Techniques that increase the permeability of the cell membrane and transfer drugs or genes to cells have been actively developed as effective therapeutic modalities. Also, in line with the development of these drug delivery techniques, the establishment of tools to verify the techniques at the cellular level is strongly required. In this study, we demonstrated an optical imaging platform integrated with an ultrasound application system to verify the feasibility of safe and efficient drug delivery through the cell membrane using ultrasound-microbubble cavitation. To examine the potential of the platform, fluorescence images of both Fura-2 AM and propidium iodide (PI) to measure calcium flux changes and intracellular PI delivery, respectively, during and after the ultrasoundmicrobubble cavitation in the cervical cancer cell were acquired. Using the optical imaging platform, we determined that calcium flux increased immediately after the ultrasound-microbubble cavitation and were restored to normal levels, and fluorescence signals from intracellular PI increased gradually after the cavitation. The results acquired by the platform indicated that ultrasound-microbubble cavitation can deliver PI into the cervical cancer cell without irreversible damage of the cell membrane. The application of an additional fluorescent imaging module and high-speed imaging modalities can provide further improvement of the performance of this platform. Also, as additional studies in ultrasound instrumentations to measure real-time cavitation signals progress, we believe that the ultrasound-microbubble cavitation-based sonoporation can be employed for safe and efficient drug and gene delivery to various cancer cells.
\end{abstract}

Keywords: optical imaging; ultrasound; microbubble; sonoporation; drug delivery; cavitation; calcium flux imaging; fluorescence imaging; Fura-2 AM; propidium iodide

\section{Introduction}

Drug delivery is a series of modalities of various physical and chemical techniques to deliver, release, and control a specific drug, so that it has an optimal effect even with a small dose [1]. Drug delivery can provide highly improved treatment efficiency for the same drug concentration, while minimizing side effects of overdoses of drugs, especially anticancer chemicals. Furthermore, drug delivery techniques that can efficiently deliver existing drugs are economically effective because developing more efficient drugs involves a lot of costs. Due to these merits in drug delivery, developments of advanced drug delivery techniques have been actively progressed using biological and chemical transporters such as a liposome [2-4], a functionalized carbon nanotube [5,6]. In addition to methods of using the transporters, studies that transfer energies such as electrical potentials [7,8] or nanoparticle-mediated light [9-11] to deliver drugs to cancer cells have been conducted. Among these drug delivery techniques, ultrasound-based drug delivery has advantages of being highly biopermeable, having high accuracy, and not permanently damaging other cells or tissues. In particular, microbubble-mediated sonoporation is a technology that can cause localized and recoverable perforation to cells or tissues, which allows drug 
delivery to targeted cells or tissue [12-18]. To be specific, ultrasound irradiation results in a microbubble oscillation, and microstreaming. The cavitation by the oscillation of the microbubble contacted on the surface of cells could change (increase) the permeability of the cellular plasma membrane [19]. The fundamental of drug delivery by the ultrasoundmicrobubble cavitation is that drugs or chemical compounds, which do not generally enter the cell, can penetrate the cell due to increased permeability by the cavitation.

For a practical use of these drug delivery techniques as a medical therapeutic modality, a platform for pre-verification of the drug delivery system is required at the in vitro cellular level. In general, a measurement of transepithelial/transendothelial electrical resistance (TEER) has been employed for monitoring drug transportation and cellular barrier changes [20-23]. However, this method does not have sufficient spatial resolution to acquire information about a single cell. On the other hand, optical imaging has the advantage of having sufficient temporal and spatial resolution while allowing noninvasive observation of a single cell. Also, in fluorescence optical imaging, fluorescent markers with different functions can be employed to acquire images of specific cellular indicators such as cell viability, calcium flux of cellular membranes, voltage potential changes.

In this study, we demonstrated an optical imaging platform integrated with an ultrasound application system for in vitro study of ultrasound-mediated drug delivery. For an application of microbubble-ultrasound cavitation to a cultured cervical cancer cell and confirmation of the potential of microbubble-assisted sonoporation for safe drug delivery, we employed the microbubble, which has been used as an ultrasound imaging contrast agent, to cervical cancer cells cultured in a cell culture flow chamber, and obtained fluorescence images of relative calcium ion $\left(\mathrm{Ca}^{2+}\right)$ concentrations and intracellular propidium iodide (PI). We also confirmed the ultrasound-induced microbubble activity (cavitation) by brightfield imaging using the platform. Information on $\mathrm{Ca}^{2+}$ flux and a gradual increase in fluorescence from intracellular PI were identified, which indicated that ultrasound-microbubble cavitation can transfer drugs into cervical cancer cells without causing irreversible cell damage. From the result of the in vitro study using the cervical cancer cells, we are convinced that the optical imaging platform integrated with an ultrasound application system can be used as a tool to effectively verify various ultrasound-based drug delivery techniques.

\section{Materials and Methods}

\subsection{Cell Culture and Sample Preparations}

We selected a cervical cancer (HeLa) cell as a target specimen because the cervical cancer cell is the most commonly employed specimen for in vitro carcinoma studies and has a high chemical/drug resistance. The cervical cancer cell line was established from American Type Culture Collection (ATCC, Manassas, VA, USA). The cervical cancer cells were cultured in $25 \mathrm{~mm}^{2}$ T-flask (Thermo Fisher Scientific, Inc., San Jose, CA, USA) using Dulbecco Modified Eagle Medium (DMEM; Invitrogen, Carlsbad, CA, USA) with 10\% fetal bovine serum (Invitrogen). A conventional cell culture incubator (MCO-19AIC, Panasonic, Osaka, Japan) was employed to give appropriate conditions $\left(37^{\circ} \mathrm{C}\right.$ temperature and $5 \%$ $\mathrm{CO}_{2}$ concentration) for maintaining viability of the cervical cancer cells.

To move the cervical cancer cells in a microfluidic cell culture flow chamber ( $\mu$-Slide I Luer, Ibidi GmbH, Martinsried, Germany) with a customized attachable water tank, $200 \mu \mathrm{L}$ cervical cells-culture media complex with a concentration of $4 \times 10^{5}$ cells $/ \mathrm{mL}$ was injected in the cell culture flow chamber. The cell culture flow chamber with the cervical cancer cells was flipped for the attachment of cells on the upper surface and the cells were cultured for $3 \mathrm{~h}$ in the incubator. After the cell attachment process, the chamber was re-flipped and the cell culture medium in the chamber was exchanged. Then, the chamber was stored for $24 \mathrm{~h}$ in the incubator to stabilize cultured cervical cancer cells.

To acquire fluorescence images of $\mathrm{Ca}^{2+}$ flux and its changes, Fura-2 AM (F1221, Invitrogen) was loaded to the cervical cancer cells cultured in the cell culture flow chamber. The detailed procedures were as follows. First of all, $1 \mathrm{~mL}$ of Fura-2 AM staining solution was produced by mixing $5 \mu \mathrm{L}$ of Fura- $2 \mathrm{AM}$ with a concentration of $1 \mathrm{mM}, 1 \mu \mathrm{L}$ of Pluronic 
Detergent (P6686, Invitrogen), and $995 \mu \mathrm{L}$ of DMEM without a fetal bovine serum. Next, $200 \mu \mathrm{L}$ of the staining solution was induced in the flow chamber with cervical cancer cells and stored for $1 \mathrm{~h}$ in the incubator. After the procedure of Fura-2 AM staining to the cells, the staining solution was washed out four times by phosphate-buffered saline (PBS, Invitrogen) and $200 \mu \mathrm{L}$ of the cell culture medium was supplied.

Also, we applied PI to test a feasibility estimation of drug delivery in cervical cancer cells by ultrasound-microbubble cavitation. PI is one of the fluorescent indicators of cells to evaluate cell viability and it is not permeable to cervical cancer cells because a plasma membrane of the cancer cells blocks PI binding to nucleic acids. For this reason, increasing fluorescence signals of PI indicates a sonoporation by the ultrasound-microbubble cavitation, which can deliver PI into the cervical cancer cell. PI purchased from Sigma-Aldrich (Carlsbad, CA, United States) and PI with a concentration of $5 \mu \mathrm{M}$ were employed in the culture media before experiments.

\subsection{Instrumentation of an Optical Imaging Platform and Imaging Procedures}

An optical imaging platform (as illustrated in Figure 1a) integrated with an ultrasound application system was investigated based on an inverted optical microscope (Ti-E, Nikon Corporation, Tokyo, Japan) to acquire both fluorescence images of Fura-2 AM and PI. The detailed description of each optical component according to the optical path and sequence is as follows. The excitation light of a light source (Lambda DG-4, Sutter Instrument, Inc., CA, United States) that consisted of a high-power xenon arc lamp and high-speed wavelength switching module was irradiated to the specimen through excitation filters, optical components, and an objective lens (S Plan Flour ELWD 20x/0.45, Nikon Corporation). To obtain relative $\mathrm{Ca}^{2+}$ concentrations from Fura-2 AM, there were two excitation filters applied, which allowed the excitation light to be adjusted with two central wavelengths of 340 and $380 \mathrm{~nm}$ (ET340x and ET380x, Chroma Technology, Bellows Falls, VT, USA). Also, a filter (69000x, Chroma Technology) corresponding to a fluorescence excitation band of PI to detect fluorescence signals from PI penetrated into the cervical cancer cell. Fluorescence with a central wavelength of $510 \mathrm{~nm}$, emitted from Fura-2 AM, was measured by a high-sensitivity electron multiplying charge-coupled device (EMCCD) camera (iXon 885, Andor Technology PLC, Belfast, Northern Ireland). To eliminate noise components with different wavelengths, an emission filter (ET510/80m, Chroma Technology) with a central wavelength of around $510 \mathrm{~nm}$ was employed. Likewise, another emission filter (69000m, Chroma Technology) was employed to measure the fluorescence emitted from PI permeated into the cervical cancer cell. Also, a multiband dichroic mirror (T400lp and 69000bs, Chroma Technology) was applied as the optical component separating the path of the light source and the detector. The platform was equipped with a multiaxis sample scanning stage (BioPrecision2, Ludl Electronic Products, Ltd., Hawthorne, NY, United States), and ultrasound irradiations and fluorescence imaging were set to be focused in the same area. Thus, it allowed the platform to move samples and perform ultrasound-microbubble cavitation and fluorescence imaging on cells in the same chamber or other chambers. A theoretical lateral resolution for fluorescence imaging of Fura-2 AM is $692 \mathrm{~nm}$ and the resolution for fluorescence imaging of PI is $814 \mathrm{~nm}$.

A single set including fluorescence images of Fura-2 AM for excitations at $340 / 380 \mathrm{~nm}$ and a fluorescence image of PI was acquired every $1.8 \mathrm{~s}$. An overall measurement time was $945 \mathrm{~s}$ ( -52.2 to $892.8 \mathrm{~s}$ ) when a trigger time of ultrasound irradiation was set to $0.0 \mathrm{~s}$. To determine the formation of microbubbles, brightfield images were captured prior to fluorescence image acquisition. Also, brightfield images were obtained instead of fluorescence images in a time domain of -3.6 to $1.8 \mathrm{~s}$ for the confirmation of whether microbubbles attached to the membrane of the cervical cancer cell cavitated after ultrasound irradiation. A white light illuminator (pE-100, CoolLED Ltd., Andover, UK) mounted above the specimen was applied as a light source to acquire brightfield images. 
(a)

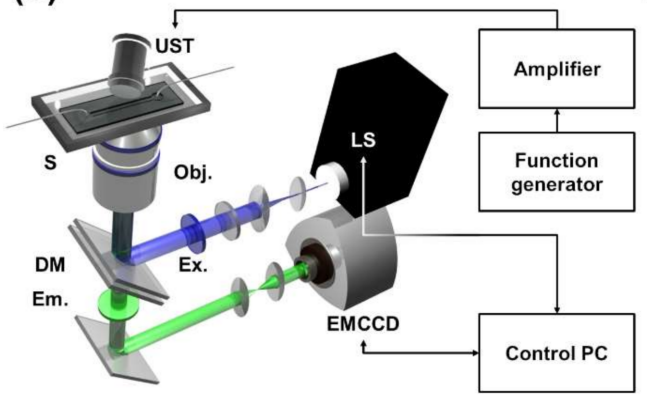

(b)
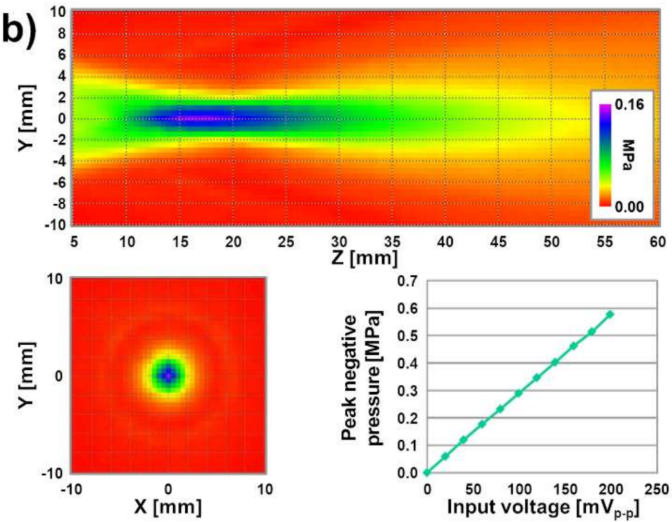

Figure 1. (a) Schematic of the optical imaging platform integrated with an ultrasound application system for obtaining fluorescence images of calcium flux changes and propidium iodide (PI) in a cervical cancer cell treated by ultrasound-microbubble cavitation. The meaning of each abbreviation in this figure is as follows. UST: an ultrasound transducer; S: a specimen that consists of a microfluidic cell culture flow chamber and a water tank; Obj.: an objective lens; DM: a dichroic mirror; Ex.: an excitation filter; Em.: an emission filter; LS: a light source for fluorescence excitation; EMCCD: an electron multiplying charge-coupled device camera. (b) Acoustic pressure fields ( $x y$ and $y z$-axis) at an input voltage of $50 \mathrm{mV}_{\mathrm{p}-\mathrm{p}}$ and a graph of peak negative acoustic pressures via input voltages of a focused ultrasound transducer.

A semi-automated operation of the optical imaging platform, brightfield/fluorescence image sequence acquisition, preprocessing, and postprocessing of acquired images were progressed by MetaMorph ${ }^{\circledR}$ (Molecular Devices, Inc., Downingtown, PA, United States). Also, we applied GNU Octave to additional postprocessing of fluorescence images and quantitative data analysis.

\subsection{Setup of an Ultrasound Application System for Ultrasound-Microbubble Cavitation}

To irradiate ultrasound and generate ultrasound-microbubble cavitation in the microfluidic cell culture flow chamber, an ultrasound application system was established. The ultrasound irradiation platform consisted of a focused immersion ultrasound transducer (A303S, Olympus NDT, Center Valley, PA, USA) with a central frequency of $1.0 \mathrm{MHz}$, an amplifier (1040L, Electronics \& Innovation, Rochester, NY, USA) with a power gain of $53 \mathrm{~dB}$, and a function generator (33220A, Agilent, Santa Clara, CA, USA) to drive the transducer.

To estimate an experimental focal length of the focused ultrasound transducer, a profile of pressures generated by the transducer was measured by an acoustic intensity measurement system (AIMS III, Onda Corporation, Sunnyvale, CA, USA) and a hydrophone (HGL-400, Onda Corporation). As described in Figure 1b, The measured center focal distance was $17 \mathrm{~mm}$ from the surface of the transducer, and the generated peak negative pressure for the focal region was $0.6 \mathrm{MPa}$ at $200 \mathrm{mV}_{\mathrm{p}-\mathrm{p}}$ of an input voltage. Based on the profile data and an optimization of the input voltage that produces a level of ultrasound that induces cavitation and does not change the position of the microbubble itself, we applied $200 \mathrm{mV}_{\mathrm{p}-\mathrm{p}}, 10$ cycles, and a single trigger as ultrasound irradiation conditions. To precisely match the focal region with the plane in which the cervical cancer cells and microbubbles were located, we employed multiaxis microscale stages that can accurately move the ultrasound transducer to the lateral $(x y)$ and axial $(z)$ axis. Also, we gave a constant angle to approach the transducer to the specimen for ensuring that the transducer did not overlap optical paths in an optical fluorescence imaging system. For this issue, additional rotation stage was applied to precise position adjustments of the ultrasound transducer. Multiaxis translational stages, the rotation stage, and mechanical components for accurate position adjustments of the ultrasound transducer were purchased from Science Town (Incheon, Republic of Korea) and Nam-il Optical Instruments Co. (Incheon, Republic of Korea). 
To effectively deliver ultrasonic waves to the cervical cancer cells in the cell culture flow chamber with water medium, a small water tank was customized using transparent acrylics. The underside of the water tank was drilled to fit the size of the flow chamber. After that, the water tank and the flow chamber were attached using a waterproof tape (Ace Cross 071, Koyo Kagaku, Tokyo, Japan) so that the acrylic of the water tank did not cover the imaging area. So, a reduction of image quality from the thick acrylic that formed the water tank was prevented.

To generate ultrasound-microbubble cavitation for calcium channel activation and drug release in the cervical cancer cell, DEFINITY ${ }^{\circledR}$ (Lantheus Medical Imaging, N. Billerica, MA, United States), an ultrasound image contrast agent, was applied with injection of PI solution after seeding of cells in the cell culture flow chamber and staining by Fura-2 AM. To be specific, $10 \mathrm{~mL}$ of a solution consisting of PI and the cell culture medium was mixed with $10 \mu \mathrm{L}$ of DEFINITY ${ }^{\circledR}$ solution, and it was injected into the flow chamber. By observing brightfield images during the access of microbubbles to the cervical cancer cell by the syringe and prior to ultrasound exposures, we confirmed sufficient approaches of microbubbles and cervical cancer cells. As shown in Figure 2, the cavitation of the microbubbles by ultrasound was observed in longitudinal brightfield images.
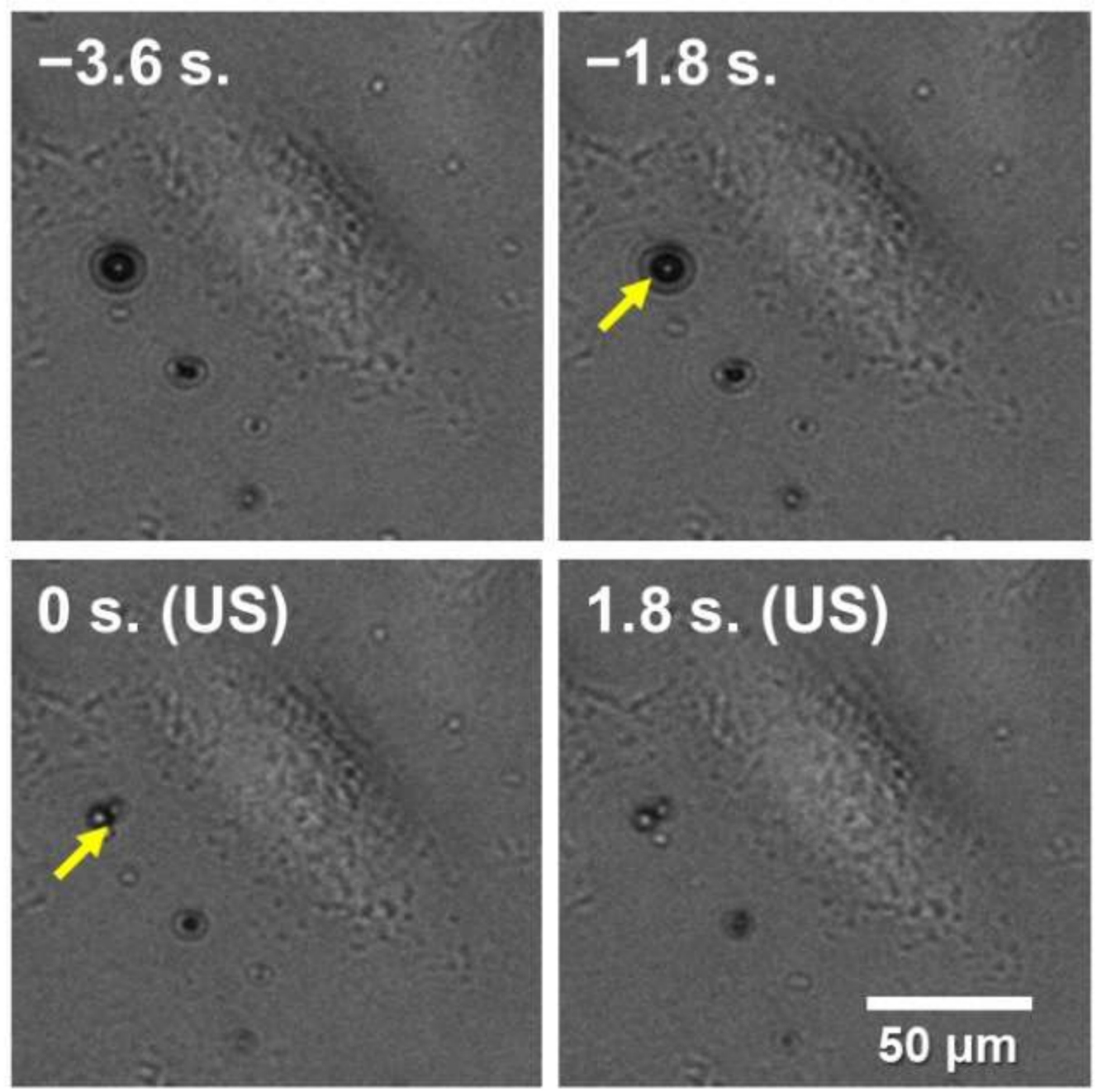

Figure 2. Brightfield images of a cavitation of microbubbles attached to a membrane of a cervical cancer cell. When comparing brightfield images before $(t=-1.8 \mathrm{~s}$.) and immediately after $(\mathrm{t}=0.0 \mathrm{~s}$. $)$ ultrasound exposures, it was observed that the size of the microbubble (indicated by a yellow arrow) was reduced due to the ultrasound-microbubble cavitation. 


\section{Results and Discussion}

\subsection{Imaging of Calcium Flux Changes of a Cervical Cell by Ultrasound-Microbubble Cavitation}

As described in Figure 2, the cavitation between ultrasound and the microbubble in contact with the cell membrane causes cracks in the plasma membrane of the cell, resulting in changes in the permeability of the cell membrane [24,25]. This increase in cell plasma membrane permeability cause changes in calcium flux in the short term and allows the entry of chemicals that cannot enter living cancer cells generally. To measure transient variations of intracellular calcium flux, fluorescence images of Fura-2 AM excited by light with a wavelength of 340 and $380 \mathrm{~nm}$ were acquired using the optical imaging platform integrated with the ultrasound application system. Relative calcium flux changes in the cervical cancer cell, which was attached to the microbubble, were obtained by calculating images of the ratio $\left(I_{\lambda}=340 \mathrm{~nm} / I_{\lambda}=380 \mathrm{~nm}\right)$ of two fluorescence images as described in Figure 3a. We confirmed that the investigated optical imaging platform provides timevarying calcium flux changes in a single cell. In images of calcium flux changes over time based on ultrasound irradiation, the ultrasound-induced microbubble cavitation offered a significant increase in the intracellular calcium concentration in the cervical cancer cell. This increase in calcium flux gradually decreased and lasted for about 50 to $60 \mathrm{~s}$ after the ultrasound-induced microbubble cavitation. After $120 \mathrm{~s}$, it recovered to a similar level of intracellular calcium concentration distributions as the image before the ultrasound exposure $(t=-1.8 \mathrm{~s})$.

(a)
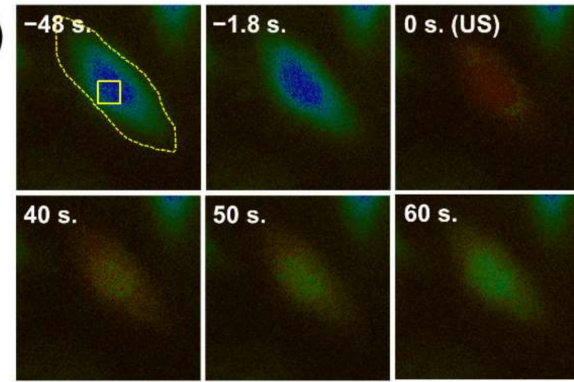

(b)
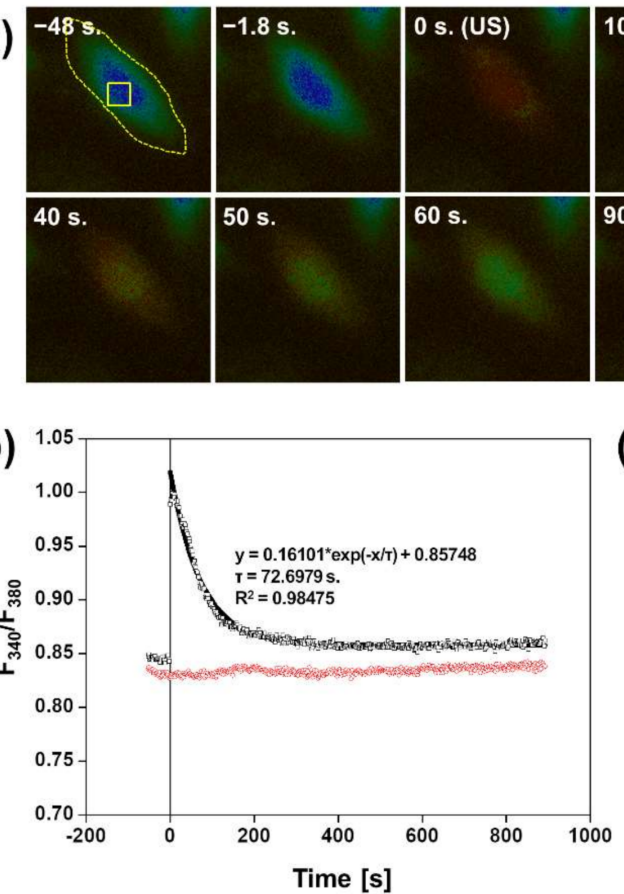

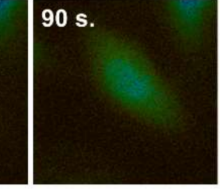

(c)

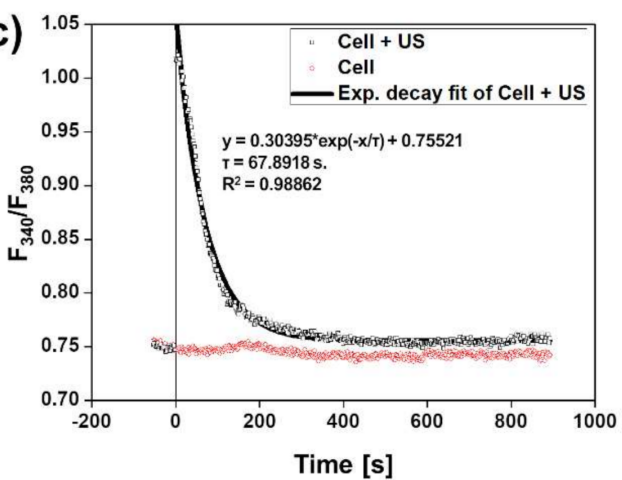

Figure 3. (a) Time-elapsed images of a ratio $\left(I_{\lambda}=340 \mathrm{~nm} / I_{\lambda}=380 \mathrm{~nm}\right)$ between fluorescence images of Fura-2 AM excited at a wavelength of 340 and $380 \mathrm{~nm}$. Background signals were corrected in each excitation wavelength. A criterion of $0.0 \mathrm{~s}$ is when ultrasound was irradiated. An inlet yellow box indicates a region of interest to determine quantitative ratios that mean calcium flux of the cervical cancer cell with the ultrasound-microbubble cavitation. $(\mathbf{b}, \mathbf{c})$ Plots of quantitative ratios between fluorescence of Fura-2 AM in the cervical cancer cell with the ultrasound-microbubble cavitation and the cell without the cavitation. Figure $3 \mathrm{~b}$ means the ratio for the entire region of cells, and Figure $3 \mathrm{c}$ indicates the ratio for a region of interest, which is represented as a yellow box, of the neighboring region in contact with the microbubble.

We determined the ratio of fluorescence by excitation lights at 340 and $380 \mathrm{~nm}$ for an entire region of the cell as described in Figure $3 \mathrm{~b}$. Also, we set a region of interest 
(yellow box in Figure 3a) for a specific region of the cell adjacent to the microbubble, and the estimation of fluorescent signals for the region of interest (ROI) was performed. A quantitative ratio analysis (as described in Figure $3 b, c$ ) in the entire region of the cell and the selected region of interest within the cervical cancer cell also indicated that the intracellular calcium concentration was recovered to its previous level before ultrasound exposures with a decaying temporal constant of 72.6979 and $68.8918 \mathrm{~s}$ following an increase in calcium flux. There is little difference between the two temporal constants, which means that the calcium flux by the ultrasound-microbubble cavitation reacts and recovers over the entire cell domain.

\subsection{Fluorescence Imaging of Propidium Iodide}

Figure $4 \mathrm{a}$ is a result of time-elapsed fluorescence images, which were acquired by the optical imaging platform with the ultrasound application system, for the detection of the increase in intracellular PI to confirm a possibility of drug delivery in the cervical cancer cell. Background fluorescence signals were corrected in each transient fluorescence image, and the detailed steps are as follows. A region without cells was set and an average of background intensities was calculated for the determined background area. After that, background correction was performed by subtracting the average value from the intensities of the acquired fluorescence images. Unlike the rapid increase in calcium flux immediately after the ultrasound exposure, the gradual increase in the fluorescence from intracellular PI was confirmed by fluorescence images of each lapsed time.

(a)
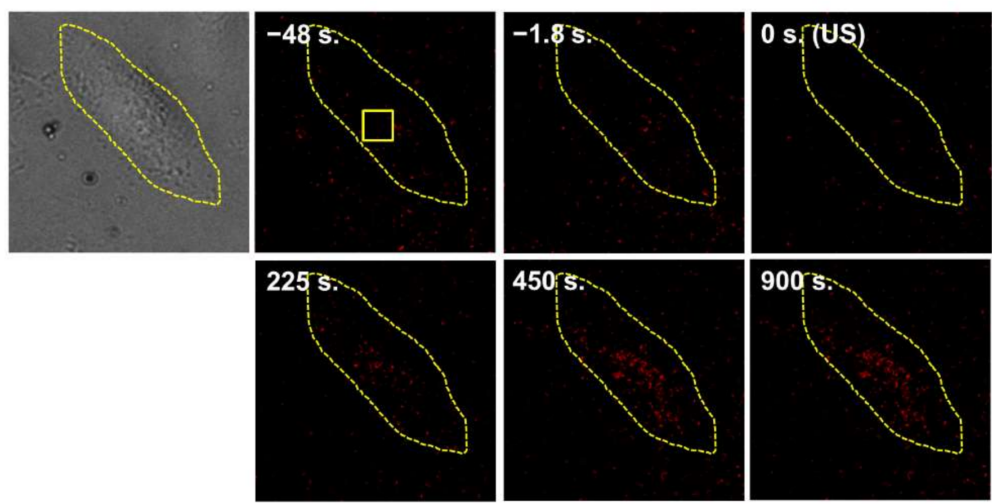

MAX
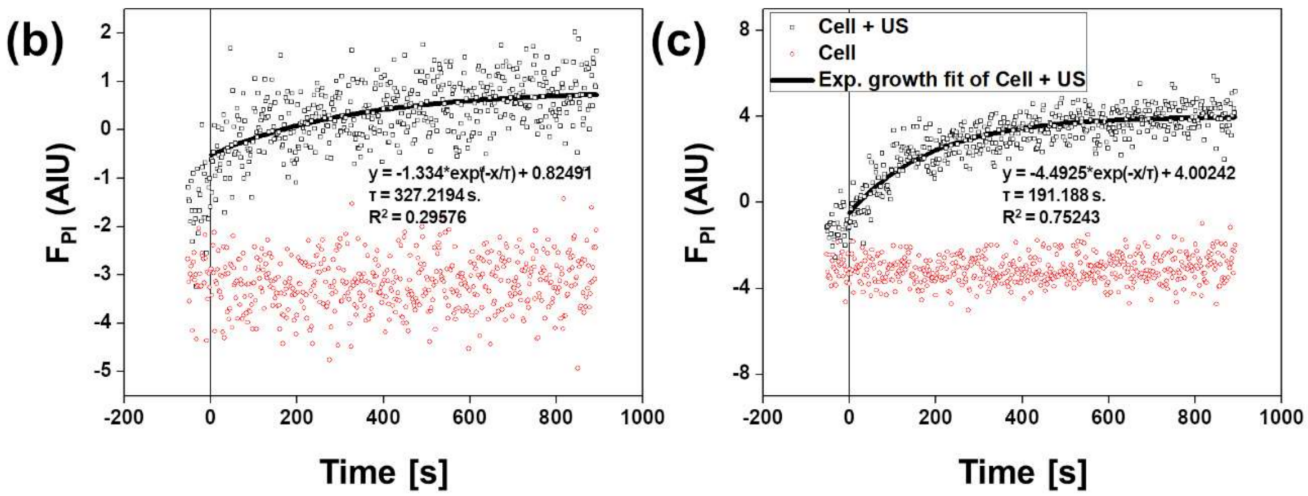

Figure 4. (a) Time-varying fluorescence images of intracellular PI in a cervical cancer cell with an ultrasound-microbubble cavitation. Background fluorescence signals were corrected. An inlet yellow box indicates a region of interest to determine intracellular PI fluorescence of the cervical cancer cell with the ultrasound-microbubble cavitation. $(\mathbf{b}, \mathbf{c})$ Plots of quantitative intensities of fluorescence from intracellular PI in the cervical cancer cell with the ultrasound-microbubble cavitation and the cell without the cavitation. Figure $3 b$ indicates the quantitative intensities of intracellular PI for the entire region of cells, and Figure $3 \mathrm{c}$ means the intracellular PI intensities for a region of interest, which is represented as a yellow box, of the neighboring region in contact with the microbubble. 
To determine the quantitative characteristics of the increasing tendency of intracellular PI, fluorescence intensities in time were estimated from a selected region of interest (yellow box in Figure 4a) within the cervical cancer cell. The result is described in Figure 4b,c. For the result to be qualitatively determined in the fluorescence images, fluorescence signals from intracellular PI were gradually increased compared to the cervical cancer cell with no cavitation. We applied an exponential growth fitting to fit the increase of fluorescence signals from intracellular PI, and a growing temporal constant was $327.2194 \mathrm{~s}$ for the entire region of the cell and $191.188 \mathrm{~s}$ for the region of interest corresponding to the membrane in contact with the microbubble. The fitting curve of the fluorescence intensity of intracellular PI for the entire cell region did not have a meaningful R-square value because the average of the overall fluorescence intensity was too small. The result indicates that PI, which cannot be normally permeated into the cancer cell, entered the cell by the ultrasoundinduced microbubble cavitation. PI reacts interchangeably between bases with no sequence preference and binds to DNA (deoxyribonucleic acid) in cells. Due to its inability to pass through the membrane of living cells, PI is commonly employed as a fluorescent marker for dead cells $[14,26,27]$. When detecting dead cells using PI, the fluorescence intensity of PI in the dead cell is $10^{3}$ to $10^{4}$ times higher than the background due to binding to DNA. In this assay, although the fluorescence of intracellular PI was increased after the ultrasound-microbubble cavitation as described in Figure $4 b, c$, the increase of fluorescence intensity was lower than that of PI in dead cells because the viability of the cervical cancer cell was not varied by the cavitation. The results from calcium flux and intracellular PI fluorescence images mean that the sonoporation did not cause irreversible damage to the cervical cancer cells, but only provided a temporary opening that can be recovered after a short period of time. It can serve the purpose of minimally invasive drug delivery, which does not cause permanent changes to cells and biological tissue while delivering chemicals.

\subsection{Discussion}

In this work, we established the optical imaging platform integrated with the ultrasound application system for in vitro assays of ultrasound-mediated drug delivery. Effective observations of changes in intracellular calcium concentration and fluorescent signal changes of intracellular PI over time indicate that the platform can be used efficiently for validation of ultrasound-mediated drug delivery techniques. Using the optical imaging platform integrated with the ultrasound application system, we derived the ultrasoundbased cavitation of a microbubble attached to a membrane of a cervical cancer cell, and obtained longitudinal fluorescence images of calcium flux and intracellular PI uptake to explore the feasibility of effective and noninvasive drug or gene delivery in cervical cancer by microbubble-mediated sonoporation. Also, it is confirmed that chemicals such as anticancer drugs can be introduced into the cervical cancer cell by the ultrasound-microbubble cavitation by observing the gradual increase of fluorescence signals from intracellular PI after the induction of the ultrasound-microbubble cavitation. As the result indicates, we confirmed that there is a significant difference between the decaying temporal constant of calcium flux (=67.8918 s.) and the growing temporal constant of fluorescence from intracellular PI (=191.188 s.), which implies a temporal mismatch between a membrane recovery for calcium ion channels and a closure of pores for drug delivery. (In this study, the time mismatch was about $2 \mathrm{~min}$ (=123.296 s.) for the region of interest corresponding to the neighboring region in contact with the microbubble.) It is correlated to the result of temporal mismatches in prior studies [14] related to drug delivery using ultrasonic cavitation, and to derive meaningful biophysical information, it is important to obtain standardized temporal constants in a specific cell line through measurements on a large number of cells. In addition, the temporal constants of intracellular PI differed scientifically in the entire region of the cell and the region of interest corresponding to the membrane region approached by the microbubble. Further studies in membrane recovery dynamics based on the temporal mismatch are expected to provide important information on drug delivery and cell therapy by ultrasound-microbubble cavitation. In particular, comparative studies 
are needed of important parameters from each cancer cell to apply ultrasound-microbubble cavitation to other carcinomas, since the cellular dynamics of carcinoma in various regions are different, and the established platform is a useful tool in the comparative studies.

Improvements that can provide higher performance in the optical imaging platform with the ultrasound application system are as follows. Currently, the optical imaging platform obtains fluorescence images to measure intracellular calcium flux and to confirm intracellular PI uptake. The system can adopt an additional fluorescent indicator to measure other cellular properties such as cell viability [28,29]. In particular, we believe that it is necessary to observe cell growth by adding additional fluorescent channels to this platform for verification of changes in long-term cell growth during and after ultrasound-microbubble cavitation. Although the temporal resolution of measuring each image sequence was slightly reduced, we are convinced that the additional implementation provides valuable information to understand cellular properties and dynamics of drug delivery. Currently, one image sequence is measured in approximately $1.8 \mathrm{~s}$ since two filter sets to measure three fluorescence images are operated using electrically operated turrets, so there is a time loss caused by switching. When only calcium imaging or PI imaging is performed individually, the overall measurement time can be further improved. In addition, each image sequence acquisition time can be improved by pixel-to-pixel binning, a use of objective lenses with higher numerical aperture, an installation of a multiband fluorescence filter set for eliminating time consumption by changing filter sets, and an application of a high-speed camera designed for voltage potential measurements in an animal brain.

In this study, DEFINITY ${ }^{\circledR}$, which is most widely employed as an ultrasound imaging contrast agent, was applied to the induction of the ultrasound-microbubble cavitation, and the use of functionalized microbubbles is believed to open a possibility of high-performance drug delivery. For instance, several studies of target binders designed for nanoparticles for the attachment of specific, targeted cancer cells have been actively progressed [30,31], and an implementation of these target binders to the microbubble can further improve selectivity as the functionalized microbubble induces the cavitation and drug delivery to selected cancer cells.

Also, it is expected that parametric studies exploring conditions appropriate for ultrasound-microbubble cavitation by applying different conditions of ultrasonic probes will provide meaningful information for the practical use of sonoporation-based drug/gene delivery. The established platform has the appropriate conditions to conduct these comparative studies.

\section{Conclusions}

In this study, we established the optical imaging platform integrated with the ultrasound application system to verify the ultrasound-induced microbubble cavitation for drug delivery to the cultured cervical cancer cell in the microfluidic cell culture flow chamber. Using the optical imaging platform, we obtained fluorescence images of both Fura-2 AM and PI to measure intracellular calcium flux changes and intracellular PI delivery during and after the ultrasound-induced microbubble cavitation. Calcium flux increased immediately after the ultrasound irradiation and recovered to normal levels with a decaying temporal constant of $67.8918 \mathrm{~s}$ for the region of interest corresponding to the neighboring region in contact with the microbubble. Unlike the trend of changing calcium flux, fluorescence signals from intracellular PI increased gradually with a growing temporal constant of $191.188 \mathrm{~s}$ for the region of interest. Through this in vitro study of ultrasound-induced microbubble cavitation, we are convinced that the optical imaging platform integrated with the ultrasound application system has adequate performance to understand drug delivery in cultured cells. Also, a possibility that the cavitation can be utilized as a technique for drug delivery without compromising the cell membrane was secured. 
Author Contributions: J.-r.C. and J.P. designed the experiment. J.-r.C. investigated an optical imaging platform to acquire fluorescence images of Fura-2 AM and intracellular PI. J.P. established an ultrasound application system and applied to the platform. J.-r.C. and J.P. performed the experiment. J.-r.C. and J.P. wrote the manuscript. All authors have read and agreed to the published version of the manuscript.

Funding: This work was supported by Original Technology Research Program for Brain Science through the National Research Foundation of Korea (NRF) funded by the Ministry of Science, ICT \& Future Planning (NRF-2016M3C7A1913933). Also, this research was supported by Innovative MD/PhD Collaborative Research Program through the National Research Foundation of Korea (NRF) funded by the Ministry of Science, ICT \& Future Planning (NRF-2019M3E5D1A02069399).

Institutional Review Board Statement: Not applicable.

Informed Consent Statement: Not applicable.

Data Availability Statement: The data presented in this study are available on request from the corresponding author.

Acknowledgments: J.-r.C. and J.P. acknowledge Hwa-Youn Lee for valuable guidance of cervical cell cultures and fluorescent indicator applications. Also, J.-r.C. and J.P. acknowledge HongSeok Cho for providing support to use the ultrasound application system.

Conflicts of Interest: The authors declare no conflict of interest.

\section{References}

1. Tiwari, G.; Tiwari, R.; Sriwastawa, B.; Bhati, L.; Pandey, S.; Pandey, P.; Bannerjee, S.K. Drug delivery systems: An updated re-view. Int. J. Pharm. Investig. 2012, 2, 2-11. [CrossRef]

2. Cao, D.; Zhang, X.; Akabar, M.D.; Luo, Y.; Wu, H.; Ke, X.; Ci, T. Liposomal doxorubicin loaded PLGA-PEG-PLGA based thermogel for sustained local drug delivery for the treatment of breast cancer. Artif. Cells Nanomed. Biotechnol. 2019, 47, 181-191. [CrossRef] [PubMed]

3. Jose, A.; Ninave, K.M.; Karnam, S.; Venuganti, V.V.K. Temperature-sensitive liposomes for co-delivery of tamoxifen and imatinib for synergistic breast cancer treatment. J. Liposome Res. 2018, 29, 153-162. [CrossRef]

4. Belhadj, Z.; Ying, M.; Cao, X.; Fu, X.; Zhan, C.; Wei, X.; Gao, J.; Wang, X.; Yan, Z.; Lu, W. Design of Y-shaped targeting mate-rial for liposome-based multifunctional glioblastoma-targeted drug delivery. J. Control. Release 2017, 255, 132-141. [CrossRef] [PubMed]

5. Liu, Z.; Chen, K.; Davis, C.; Sherlock, S.; Cao, Q.; Chen, X.; Dai, H. Drug delivery with carbon nanotubes for in vivo cancer treatment. Cancer Res. 2008, 68, 6652-6660. [CrossRef]

6. Mehdipoor, E.; Adeli, M.; Bavadi, M.; Sasanpour, P.; Rashidian, B. A possible anticancer drug delivery system based on car-bon nanotube-dendrimer hybrid nanomaterials. J. Mater. Chem. 2011, 21, 15456-15463. [CrossRef]

7. Bhutiani, N.; Agle, S.; Li, Y.; Li, S.; Martin, R.C. Irreversible electroporation enhances delivery of gemcitabine to pancreatic adenocarcinoma. J. Surg. Oncol. 2016, 114, 181-186. [CrossRef]

8. Kulbacka, J.; Daczewska, M.; Dubińska-Magiera, M.; Choromańska, A.; Rembiałkowska, N.; Surowiak, P.; Kulbacki, M.; Kotulska, M.; Saczko, J. Doxorubicin delivery enhanced by electroporation to gastrointestinal adenocarcinoma cells with P-gp overexpression. Bioelectrochemistry 2014, 100, 96-104. [CrossRef] [PubMed]

9. Liu, J.; Bu, W.; Pan, L.; Shi, J. NIR-triggered anticancer drug delivery by upconverting nanoparticles with integrated azo-benzenemodified mesoporous silica. Angew. Chem. 2013, 52, 4375-4379. [CrossRef] [PubMed]

10. Qin, Y.; Chen, J.; Bi, Y.; Xu, X.; Zhou, H.; Gao, J.; Hu, Y.; Zhao, Y.; Chai, Z. Near-infrared light remote-controlled intracellular anti-cancer drug delivery using thermo/pH sensitive nanovehicle. Acta Biomater. 2015, 17, 201-209. [CrossRef] [PubMed]

11. Wang, S.; Zhao, X.; Wang, S.; Qian, J.; He, S. Biologically inspired polydopamine capped gold nanorods for drug delivery and light-mediated cancer therapy. ACS Appl. Mater. Interfaces 2016, 8, 24368-24384. [CrossRef] [PubMed]

12. Wu, J.; Pepe, J.; Rincon, M. Sonoporation, anti-cancer drug and antibody delivery using ultrasound. Ultrasonics 2006, 44, e21-e25. [CrossRef]

13. Maeda, H.; Tominaga, K.; Iwanaga, K.; Nagao, F.; Habu, M.; Tsujisawa, T.; Seta, Y.; Toyoshima, K.; Fukuda, J.-I.; Nishihara, T. Targeted drug delivery system for oral cancer therapy using sonoporation. J. Oral Pathol. Med. 2009, 38, 572-579. [CrossRef] [PubMed]

14. Fan, Z.; Kumon, R.E.; Park, J.; Deng, C.X. Intracellular delivery and calcium transients generated in sonoporation facilitated by microbubbles. J. Control. Release 2010, 142, 31-39. [CrossRef]

15. Cho, H.; Lee, H.-Y.; Han, M.; Choi, J.-R.; Ahn, S.; Lee, T.; Chang, Y.; Park, J. Localized down-regulation of P-glycoprotein by focused ultrasound and microbubbles induced blood-brain barrier disruption in rat brain. Sci. Rep. 2016, 6, 31201. [CrossRef] [PubMed]

16. Chowdhury, S.M.; Lee, T.; Willmann, J.K. Ultrasound-guided drug delivery in cancer. Ultrasonography 2017, 36, 171-184. [CrossRef] [PubMed] 
17. Horsley, H.; Owen, J.; Browning, R.; Carugo, D.; Malone-Lee, J.; Stride, E.; Rohn, J. Ultrasound-activated microbubbles as a novel intracellular drug delivery system for urinary tract infection. J. Control. Release 2019, 301, 166-175. [CrossRef]

18. Kato, S.; Shirai, Y.; Motozono, C.; Kanzaki, H.; Mori, S.; Kodama, T. In vivo delivery of an exogenous molecule into murine T lymphocytes using a lymphatic drug delivery system combined with sonoporation. Biochem. Biophys. Res. Commun. 2020, 525, 1025-1031. [CrossRef]

19. Peruzzi, G.; Sinibaldi, G.; Silvani, G.; Ruocco, G.; Casciola, C.M. Perspectives on cavitation enhanced endothelial layer permeability. Colloids Surf. B 2018, 168, 83-93. [CrossRef]

20. Ghaffarian, R.; Muro, S. Models and methods to evaluate transport of drug delivery systems across cellular barriers. J. Vis. Exp. 2013, 80, 50638. [CrossRef] [PubMed]

21. Srinivasan, B.; Kolli, A.R.; Esch, M.B.; Abaci, H.E.; Shuler, M.L.; Hickman, J.J. TEER measurement techniques for in vitro bar-rier model systems. J. Lab. Autom. 2015, 20, 107-126. [CrossRef]

22. Tan, H.-Y.; Trier, S.; Rahbek, U.L.; Dufva, M.; Kutter, J.P.; Andresen, T.L. A multi-chamber microfluidic intestinal barrier model using Caco-2 cells for drug transport studies. PLoS ONE 2018, 13, e0197101. [CrossRef]

23. Asif, A.; Kim, K.H.; Jabbar, F.; Kim, S.; Choi, K.H. Real-time sensors for live monitoring of disease and drug analysis in microfluidic model of proximal tubule. Microfluid. Nanofluidics 2020, 24, 1-10. [CrossRef]

24. Claudio, P.P.; Howard, C.M.; Nande, R. Ultrasound-mediated oncolytic virus delivery and uptake for increased therapeutic efficacy: State of art. Oncolytic Virotherapy 2015, 4, 193-205. [CrossRef] [PubMed]

25. Lentacker, I.; De Cock, I.; Deckers, R.; De Smedt, S.; Moonen, C. Understanding ultrasound induced sonoporation: Definitions and underlying mechanisms. Adv. Drug Deliv. Rev. 2014, 72, 49-64. [CrossRef]

26. Krämer, C.E.M.; Wiechert, W.; Kohlheyer, D. Time-resolved, single-cell analysis of induced and programmed cell death via non-invasive propidium iodide and counterstain perfusion. Sci. Rep. 2016, 6, 32104. [CrossRef]

27. Crowley, L.C.; Scott, A.P.; Marfell, B.J.; Boughaba, J.A.; Chojnowski, G.; Waterhouse, N.J. Measuring cell death by propidi-um iodide uptake and flow cytometry. Cold Spring Harb. Protoc. 2016, 2016, pdb-prot087163.

28. Oh, T.; Sung, J.H.; Tatosian, D.A.; Shuler, M.L.; Kim, D. Real-time fluorescence detection of multiple microscale cell culture analog devices in situ. Cytom. A 2007, 71A, 857-865. [CrossRef] [PubMed]

29. Muslimov, A.R.; Timin, A.S.; Bichaykina, V.R.; Peltek, O.O.; Karpov, T.E.; Dubavik, A.; Nominé, A.; Ghanbaja, J.; Sukhorukov, G.B.; Zyuzin, M.V. Biomimetic drug delivery platforms based on mesenchymal stem cells impregnated with light-responsive submicron sized carriers. Biomater. Sci. 2019, 8, 1137-1147. [CrossRef] [PubMed]

30. Patitsa, M.; Karathanou, K.; Kanaki, Z.; Tzioga, L.; Pippa, N.; Demetzos, C.; Verganelakis, D.A.; Cournia, Z.; Klinakis, A. Magnetic nanoparticles coated with polyarabic acid demonstrate enhanced drug delivery and imaging properties for cancer theranostic applications. Sci. Rep. 2017, 7, 1-8. [CrossRef] [PubMed]

31. Zhang, N.; Li, M.; Sun, X.; Jia, H.; Liu, W. NIR-responsive cancer cytomembrane-cloaked carrier-free nanosystems for highly efficient and self-targeted tumor drug delivery. Biomaterials 2018, 159, 25-36. [CrossRef] [PubMed] 